\title{
Numerical model for individual time calculation for evacuation among secondary students
}

\begin{abstract}
Purpose: The purpose of this paper is to design a numerical model to calculate the individual evacuation time among secondary students based on Knowledge, Attitude and Practice (KAP), human characteristics and travel distances. Design/methodology/approach: Validated KAP questionnaires were distributed among 290 respondents. The KAP level was obtained based on the assigned scores. During a fire drill, the individual evacuation time was calculated by using personal digital watch while the travel distances were recorded and measured. A linear numerical model was derived by using multiple linear regression to identify the significant variables and the coefficients. Findings: The CVI, CVR and Cronbach's $\alpha$ value $(0.75,0.59$ and 0.7 , respectively) which are greater than minimum accepted level proved the reliability and consistency of the instrument. The evacuation time prediction by the developed numerical model showed strong correlation with the actual time $(R=0.95)$. The regression analysis found that 89 per cent proportion of variance in the evacuation time are determined by the predictors. Based on the linear equation, it found that the decrease in weight, knowledge level and walking speed while increase in BMI, flat and stair travel distances could increase evacuation time. From the six significant variables, weight, walking speed, flat and stair distances showed significant correlation in the model with $p<0.001$, while BMI and knowledge showed $p<0.05$. The integration with mobility factors expand the formula which applicable within dynamic fire scenario. Research limitations/implications: The involvement of examination students in the study is restricted by the Ministry of Education Malaysia to avoid interruption of learning session which limited the data representation. Originality/value: Instead of using the traditional direct measurement of the evacuation time, the developed numerical model is an alternative convenient approach which could be used as one of the pre-assessment tool to identify the level of safety among students. The low cost and shorter time application of this model become one of the greatest advantages compared to other available approaches. The calculated individual evacuation time could be used directly to develop a better fire safety policy.
\end{abstract}

Keyword: CVR; CVI; Evacuation time; Human charateristics; KAP; Travel distances 